\title{
Magnetic flux transfer in the 5 April 2010 Galaxy 15 substorm: an unprecedented observation
}

\author{
M. Connors ${ }^{1,2}$, C. T. Russell ${ }^{2,3}$, and V. Angelopoulos ${ }^{2,3}$ \\ ${ }^{1}$ Athabasca University Geophysical Observatory, Athabasca AB, Canada \\ ${ }^{2}$ Department of Earth and Space Sciences, UCLA, Los Angeles, CA, USA \\ ${ }^{3}$ IGPP, UCLA, Los Angeles, CA, USA
}

Received: 21 January 2011 - Revised: 10 March 2011 - Accepted: 17 March 2011 - Published: 30 March 2011

\begin{abstract}
At approximately 08:25 UT on 5 April 2010, a CME-driven shock compressed Earth's magnetosphere and applied about $15 \mathrm{nT}$ of southward IMF for nearly an hour. A substorm growth phase and localized dipolarization at 08:47 UT were followed by large dipolarizations at 09:03 UT and 09:08 UT, observed by GOES West (11) in the midnight sector, and by three THEMIS spacecraft near $X=-11$, $Y=-2 R_{\mathrm{E}}$. A large electric field at the THEMIS spacecraft indicates so much flux transfer to the inner magnetosphere that "overdipolarization" took place at GOES 11. This transfer is consistent with the ground and space magnetic signature of the substorm current wedge. Significant particle injections were also observed. The ensemble of extreme geophysical conditions, never previously observed, is consistent with the Near-Earth Neutral Line interpretation of substorms, and subjected the Galaxy 15 geosynchronous satellite to space weather conditions which appear to have induced a major operational anomaly.
\end{abstract}

Keywords. Magnetospheric physics (Electric fields; Magnetotail; Storms and substorms)

\section{Introduction}

The shock associated with the coronal mass ejection of 3 April 2010 (described in detail by Möstl et al., 2010) arrived at Earth at approximately 08:25 UT on 5 April 2010. The THEMIS A, D, and E spacecraft, with comprehensive instrument suites (Burch and Angelopoulos, 2009), were near each other in the northern plasma sheet in the early morning sector (Fig. 1), and GOES 11 was near magnetic midnight at $225.5^{\circ}$ geographic longitude. The Galaxy-15 geosynchronous communications satellite was at longitude $227.03^{\circ}$ (ucsusa.org database; updated 1 April 2010), very near GOES 11, and in

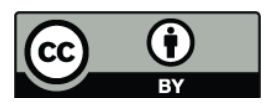

Correspondence to: M. Connors (martinc@athabascau.ca) eclipse until approximately 09:20 UT, with anomalies leading to loss of control reported $28 \mathrm{~min}$ later (Allen, 2010).

Geotail being near the Earth-Sun line at a distance of $14.7 R_{\mathrm{E}}$, only approximately $100 \mathrm{~s}$ were required to translate its solar wind observations to the magnetopause (Fig. 1c). The magnetic field behind the shock was initially slightly northward, but $B_{\mathrm{Z}}$ quickly turned to about $-13 \mathrm{nT}$. Increase of plasma density from about 4 to over 10 ions/cc, and of speed from about 550 to $700 \mathrm{~km} \mathrm{~s}^{-1}$, led to a dynamic pressure increase from about $2 \mathrm{nPa}$ to $10 \mathrm{nPa}$, and compression of the magnetosphere. After increasing, the total pressure remained relatively steady. Due to short duration of southward IMF, the resulting storm was of low intensity (Möstl et al., 2010). However, the strong southward field and high dynamic pressure in the sheath immediately following the ICME shock led to impressive short-term effects as described here.

\section{Compression}

Magnetic signals at GOES 11 and THEMIS A are shown in Fig. 1. Compression began with the arrival of the shock at 08:25 UT. The field began to stretch immediately at shock arrival, as indicated by an increase in $B_{X}$ and a decrease in $B_{\mathrm{Z}}$. At 08:47 UT, the field at GOES dipolarized, the typical signature of a substorm in this region, but not accompanied by major field changes at $-11 R_{\mathrm{E}}$. After this small substorm, not discussed in detail here, the stretching at GOES resumed, with $B_{X}$ attaining its previous highly stretched value of $100 \mathrm{nT}$ before beginning to dipolarize at 09:02 UT. At 09:03 UT, dipolarization began at THEMIS A, with initial decrease in GSM $B_{\mathrm{X}}$ from 65 to about $50 \mathrm{nT}$, recovery to over $65 \mathrm{nT}$, and decrease to below $20 \mathrm{nT}$ just after 09:08 UT. An increase in $B_{\mathrm{Z}}$, change in $B_{\mathrm{Y}}$, and more variability in the field suggest entry to the PSBL at the initiation of dipolarization. GOES 11 dipolarization had a decrease in $B_{\mathrm{X}}$ from $90 \mathrm{nT}$ to $10 \mathrm{nT}$ between 09:01 UT and 09:13 UT and

Published by Copernicus Publications on behalf of the European Geosciences Union. 

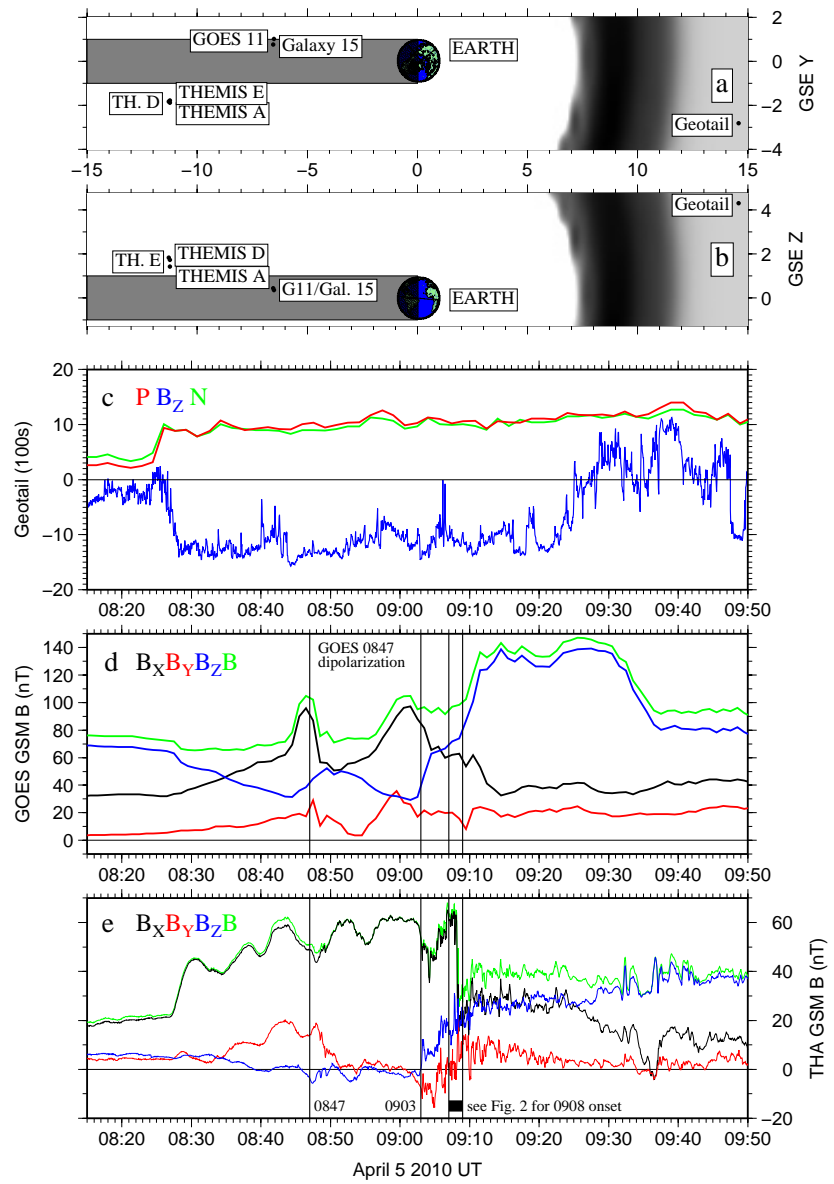

Fig. 1. Spacecraft positions and data on 5 April 2010. (a) GSE XY spacecraft positions at 08:36 UT. Magnetosheath and solar wind B roughly indicated by grey shading. (b) As in (a), in GSE XZ coordinates. (c) Geotail solar wind values (100 s delay) of GSM $B_{Z}$ in nT (blue), dynamic pressure $P$ in $\mathrm{nPa}$ (red), and proton density in $\mathrm{cm}^{3}$ (green). (d) GOES 11 magnetic field. "Overdipolarization" of the $B_{\mathrm{Z}}$ component above usual geosynchronous values lasts from 09:10 to 09:35 UT. (e) THEMIS A magnetic field in GSM coordinates.

$B_{\mathrm{Z}}$ that "overdipolarized" (a behavior earlier noted by Akasofu, 2003), attaining values of over $140 \mathrm{nT}$. Despite little change in solar wind dynamic pressure, and continued large negative IMF $B_{Z}$, which persisted until northward turning at roughly 09:30 UT, tail stretching did not resume in the period following the overdipolarization.

\section{Onsets}

The two step 09:03/09:08 UT dipolarization described above may be described in two complementary ways. Ground and space magnetic fields may be attributed to the electric currents of a substorm current wedge or SCW (McPherron et al., 1973). The increase in magnetic field in the inner magnetosphere can also be viewed as a result of flux transfer from the more distant tail. As highly conductive plasma moves in the

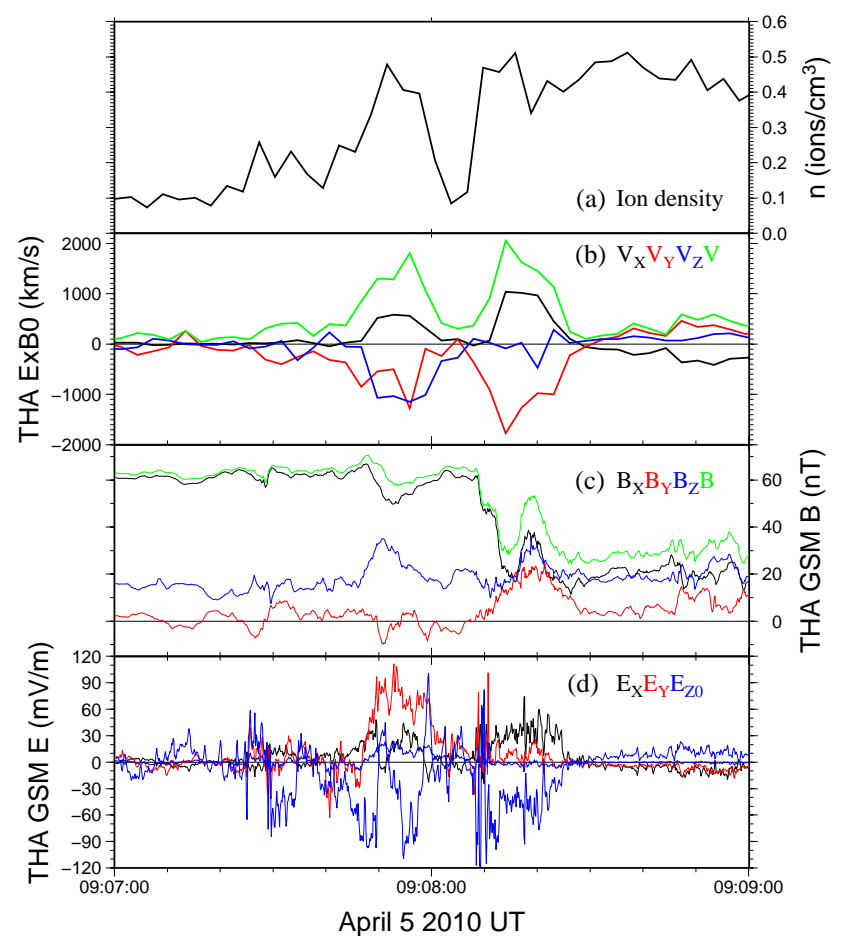

Fig. 2. THEMIS A quantities. (a) Ion density. (b) $\boldsymbol{E} \times \boldsymbol{B}$ velocity assuming $\boldsymbol{E} \cdot \boldsymbol{B}=0$. (c) GSM magnetic field. (d) GSM electric field with $E_{\mathrm{Z}}$ derived from $\boldsymbol{E} \cdot \boldsymbol{B}=0$.

observed magnetic field, an electric field is observed in the spacecraft (rest) frame. The magnetic field "frozen in" to the plasma is transported with it. The perpendicular electric field measures the flux transfer rate.

\subsection{Electric field observations}

The THEMIS spacecraft are well equipped for measurement of electric fields near the plane of the ecliptic, with spinning 50 and $40 \mathrm{~m}$ baselines, and less so perpendicular to it, with only a $6.9 \mathrm{~m}$ baseline along the spin axis (Bonnell et al., 2008). We have used the spin-plane measurements with offsets negligible compared to the size of the signal, and determined the axial signal (roughly GSM $E_{\mathrm{Z}}$ ) from them by requiring that $\boldsymbol{E} \cdot \boldsymbol{B}=0$. Dipolarization started at THEMIS at 09:03 UT, but large electric fields were observed only during the two minute time interval 09:07 to 09:09 UT shown in Fig. 2. The large decrease in $B_{\mathrm{X}}$ at 09:08:10 corresponded to recovery of the plasma sheet over the spacecraft. Two large $E_{\mathrm{Y}}$ spikes were observed then, which based on other data we conclude to be real but do not discuss further here. It was bracketed by pulses of large electric field from 09:07:40 to 09:08:00, and from 09:08:10 to 09:08:25. These both had negative $E_{\mathrm{Z}}$ components, at times exceeding $90 \mathrm{mV} \mathrm{m}^{-1}$ in magnitude. The initial pulse had a positive $E_{\mathrm{Y}}$ of comparable magnitude, while the second had $E_{\mathrm{X}}$ of about $+30 \mathrm{mV} \mathrm{m}^{-1}$. These, and the magnetic field geometry, are consistent with 


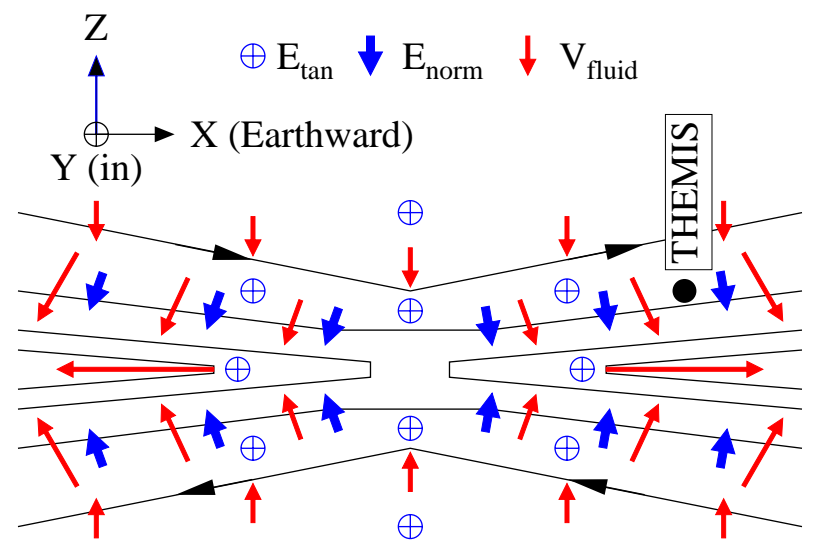

Fig. 3. Schematic based on Wygant et al. (2005) showing the region tailward of $-10 R_{\mathrm{E}}$. Electric fields are indicated in blue, plasma flows in red, and magnetic fields in black. The rough position of the THEMIS spacecraft is indicated. In keeping with the original diagram, only the in-plane fluid velocity is shown. It may be inferred from $\boldsymbol{E} \times \boldsymbol{B}$ that $\boldsymbol{V}$ has a significant out-of-page component.

observations of large perpendicular electric fields by Wygant et al. (2005), if THEMIS was situated earthward of the reconnection X-line and near the plasma sheet boundary as shown in Fig. 3. Using the frozen-in condition, the plasma velocity of $\boldsymbol{v}=\boldsymbol{E} \times \boldsymbol{B} / B^{2}$, shown in Fig. 2, with its downward and Earthward components, is also consistent with this picture. There is also a large $-Y$ velocity component. The 1000 to $2000 \mathrm{~km} \mathrm{~s}^{-1}$ magnitudes of any of these components are 5 to 10 times those of typical substorms. The $\mathrm{Y}$ velocity component, not commented on by Wygant et al. (2005), is consistent with MHD requirements, applicable as we infer that THEMIS was relatively far from the X-line. In Fig. 3 it is clear that the direction of $\boldsymbol{E} \times \boldsymbol{B}$ would be along $-Y$ (out of the page) at all points where $B$ is nonzero, but for clarity this is not shown. The $-Y$ velocity component is consistent with expansion (H. Singer, personal communication) of the center of activity toward the east, and with the location of the THEMIS spacecraft in the morning sector. However, we will assume that most flux transfer took place in the midnight sector, explaining the overdipolarization at GOES 11, and concentrate on the large positive $\mathrm{X}$ velocity component bringing flux Earthward.

We may roughly approximate the two pulses of Earthward flow near 09:08 UT as having an average speed $v_{\mathrm{X}} \sim$ $500 \mathrm{~km} \mathrm{~s}^{-1}$, lasting $\Delta t \sim 30 \mathrm{~s}$ (about $2.5 R_{\mathrm{E}}$ of passing flow), and with an average $B_{\mathrm{Z}} \sim 25 \mathrm{nT}$. If we consider that we are near the edge of a flow about $\Delta Y \sim 3 R_{\mathrm{E}}$ wide, that flow would carry $v_{\mathrm{X}} \Delta t B_{\mathrm{Z}} \Delta Y \sim 7.5 R_{\mathrm{E}}^{2} \times 25 \mathrm{nT} \sim 7 \mathrm{MWb}$ in the interval when THEMIS A could sense the flow. The total flux transported during the event would be much larger. The cited width is from Angelopoulos et al. (1994), who also give about $2.5 \mathrm{MWb}$ as typical flux transport from a single bursty bulk flow, much less than that transported in an entire substorm. The actual width is not important; rather conver-

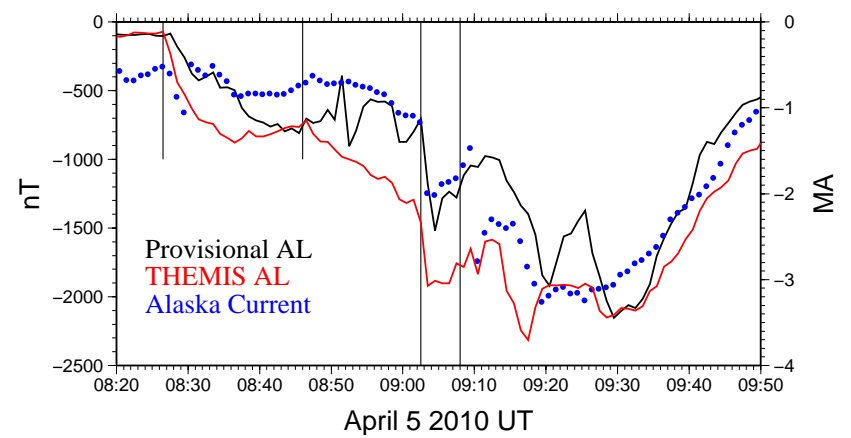

Fig. 4. Ground indices. Provisional and THEMIS AL indices (left scale), and the cross-meridian current in Alaska in MA (right scale). Vertical bars indicate the times of the shock arrival (leftmost) and the three substorm onsets described in the text.

gence of flow and compression in moving toward the inner magnetosphere is. The width decreasing by a factor near 2 , with a similar lengthwise compression in slowing down near geosynchronous orbit, could produce up to $100 \mathrm{nT}$ of additional magnetic field there. The observed overdipolarization to $140 \mathrm{nT}, 60 \mathrm{nT}$ beyond a typical nightside $80 \mathrm{nT}$, is readily explained by flux transport based on observed electric and magnetic fields. However, this field enhancement can only be maintained by a strong flow from the tail, of dynamic pressure comparable to that of the solar wind on the front side, i.e. about $8 \mathrm{nPa}$. This flow must persist about $20 \mathrm{~min}$. As shown in Fig. 1d, after initial dipolarization at GOES 11 back to the typical $80 \mathrm{nT}$ value by 09:09 UT, overdipolarization followed the flux transfer, the correct temporal relationship. The 20-min overdipolarization requires the flow to last 40 times longer than the observed flow burst at THEMIS A. Thus the transferred flux was probably about $300 \mathrm{MWb}$. This is about $1 / 3$ of the flux stored in the tail, but it was actively replenished by dayside reconnection, so the net reduction was less. At 09:30 UT, consistent with the overdipolarization relaxing at that time, the flow probably ceased. We now further show that overdipolarization is consistent with the current flowing in the SCW at this time.

\subsection{Ground observations}

Figure 4 shows the THEMIS AL index (Angelopoulos et al., 2009), from a relatively dense network of North American stations, the provisional AL index, derived from fewer and more sparsely distributed stations, and the cross-meridan current derived from the magnetometer chain of the University of Alaska, inverted using an automated process based on models of Kisabeth and Rostoker (1977). Inversion was checked by comparison with the original magnetograms, which it represents well. This third indicator of auroral currents has the current magnitude plotted on a scale facilitating comparison to the AL indices.

After responding to the compression, but not much to the initial onset at 08:47 UT, all three indices showed the 09:03 
and 09:08 UT onsets. The THEMIS AL index had a stronger response to the first of these, likely due to the auroral electrojets having moved equatorward into a region where it has dense coverage. The 09:08 UT onset, for which we consider flux transfer, was responded to with a short time delay by THEMIS AL and by the inversion results, while standard $\mathrm{AL}$ responded less, and later. We attribute this to the sparse station coverage by this index. From midlatitude station $\mathrm{Y}$ (eastward) component ground perturbations (not shown), we infer that downward field-aligned currents (FAC) of the SCW were east of Alaska and the meridian of GOES 11 after the 09:08 UT onset, and that the upward currents were over Asia. This is consistent with the strongest westward electrojet signature (from the SCW ionospheric closure current) at this time being in Alaska. The SCW current was of maximum total strength about $3 \mathrm{MA}$ as inferred from inversion. Much as an $\mathrm{AL}$ of -2000 is much larger in magnitude than is typical of substorms, this current is very large for the SCW. The northward perturbation at GOES 11 that would result if $3 \mathrm{MA}$ of current followed the field lines of a maximally stretched Tsyganenko 89 model, with FAC longitudes in western North America and Asia, is $40 \mathrm{nT}$. This is comparable to the observed amount of overdipolarization observed at GOES 11, beyond normal values at GEO.

\section{Conclusions}

The space weather event of 5 April 2010 arose from the sheath of a CME impacting Earth, significantly enhancing an already southward IMF. After the shock, a growth phase ensued which was not much diminished by a first substorm onset at 08:47 UT. A larger double onset took place at 09:03/09:08 UT, in the latter of which significant Earthward flux transfer took place as suggested by electric fields observed at THEMIS. The field changes at THEMIS are consistent with its position relative to the $\mathrm{X}$-line, and we note large transverse fields consistent with those observed (likely closer to the X-line) by Wygant et al. (2005). We note that the geometry implies large $-Y$ flow velocities, which were observed. The Earthward component of flux transfer manifested in the inner magnetosphere as "overdipolarization" signatures at GOES 11 in the midnight sector. These in turn are consistent with the current determined to flow in the $\mathrm{SCW}$ as deduced quantitatively from electrojet signatures in Alaska. Space does not permit description of particle data indicating an injection of both protons and electrons of a magnitude consistent with the abnormally large field signatures we have described. Allen (2010) indicates that Galaxy 15 was in a vulnerable near-midnight position during a period with extreme AE values at the time of failure (09:48 UT). We have documented in-situ field data that is similarly extreme, along with verification of the magnitude of the SCW current that is causative of AE. In the matter of the Galaxy 15 failure, we cannot specify a direct cause, but note that unusually active conditions had prevailed for more than an hour before it. It indeed seems likely these unsettled conditions were a factor in the Galaxy 15 anomaly.

Acknowledgements. T. Nagai supplied Geotail MGF data, and $\mathrm{X}$. Chu computed the THEMIS AE-index. Geotail EPIC and CPI data were obtained from CDAWeb courtesy of D. Williams and L. Frank, respectively. We acknowledge NASA contract NAS5-02099, J. W. Bonnell and F. S. Mozer for use of EFI data, K.-H. Glassmeier for FGM data, H. J. Singer for GOES 11 data, and the University of Alaska for meridian chain data.

Topical Editor R. Nakamura thanks one anonymous referee for her/his help in evaluating this paper.

\section{References}

Akasofu, S.: A source of auroral electrons and the magnetospheric substorm current systems, J. Geophys. Res. (Space Physics), 108, 8006, doi:10.1029/2002JA009547, 2003.

Allen, J.: The Galaxy 15 Anomaly: Another Satellite in the Wrong Place at a Critical Time, Space Weather, 8, 2, doi:10.1029/2010SW000588, 2010.

Angelopoulos, V., Kennel, C. F., Coroniti, F. V., Pellat, R., Kivelson, M. G., Walker, R. J., Russell, C. T., Baumjohann, W., Feldman, W. C., and Gosling, J. T.: Statistical characteristics of bursty bulk flow events, J. Geophys. Res., 99, 21257-21280, 1994.

Angelopoulos, V., McFadden, J. P., Larson, D., Carlson, C. W., Mende, S. B., Frey, H., Phan, T., Sibeck, D. G., Glassmeier, K.H., Auster, U., Donovan, E., Mann, I. R., Rae, I. J., Russell, C. T., Runov, A., Zhou, X.-Z., and Kepko, L.: Response to Comment on Tail Reconnection Triggering Substorm Onset, Science, 324, 1391-c, doi:10.1126/science.1168045, 2009.

Bonnell, J. W., Mozer, F. S., Delory, G. T., Hull, A. J., Ergun, R. E., Cully, C. M., Angelopoulos, V., and Harvey, P. R.: The Electric Field Instrument (EFI) for THEMIS, Sp. Sci. Rev., 141, 303341, 2008.

Burch, J. L. and Angelopoulos, V. (Ed.): The THEMIS Mission, Springer, New York, doi:10.1007/978-0-387-89820-9, 2009.

Kisabeth, J. L. and Rostoker, G.: Modelling of three-dimensional current systems associated with magnetospheric substorms, Geophys. J. Int., 49, 655-683, 1977.

McPherron, R. L., Russell, C. T., and Aubry, M. P.: Satellite studies of magnetospheric substorms on August 15, 1968. 9. Phenomenological model for substorms., J. Geophys. Res., 78, 3131-3149, 1973.

Möstl, C., Temmer, M., Rollett, T., Farrugia, C. J., Liu, Y., Veronig, A. M., Leitner, M., Galvin, A. B., and Biernat, H. K.: STEREO and Wind observations of a fast ICME flank triggering a prolonged geomagnetic storm on 5-7 April 2010, Geophys. Res. Lett., 37, 24103, doi:10.1029/2010GL045175, 2010.

Wygant, J. R., Cattell, C. A., Lysak, R., Song, Y., Dombeck, J., McFadden, J., Mozer, F. S., Carlson, C. W., Parks, G., Lucek, E. A., Balogh, A., Andre, M., Reme, H., Hesse, M., and Mouikis, C.: Cluster observations of an intense normal component of the electric field at a thin reconnecting current sheet in the tail and its role in the shock-like acceleration of the ion fluid into the separatrix region, J. Geophys. Res. (Space Physics), 110, 9206, doi:10.1029/2004JA010708, 2005. 